\title{
Urkunden und Zeugen im Rechtsleben des Hochmittelalters
}

\section{Charters and witnesses in the law practice of the high middle Ages}

Reinhard Härtel / reinhard.haertel@uni-graz.at

Institut für Geschichte, Universität Graz

\begin{abstract}
Abstract: The aim of the article is to clarify the use of charters in the high middle ages, not according to their production but with reference to the formulae and the practical statements listed in them. Most of the named examples derive from Austria and Slovenia. Charters are often confirmed, but there is only little evidence of their use as a proof in court. The issuers were often afraid, their legal successors wouldn't recognise their legal acts. Confirmations assured their permanence and the persistence of witnesses. The general knowledge of the legal act protected it somehow, but malicious infringers couldn't be turned away otherwise than through witnesses. The charters were mere reminders of the legal act itself and not a kind of protection. Witnesses obtained their knowledge about legal acts more often from legal charters than indicated by the historical tradition.
\end{abstract}

\section{Keywords}

Use of charters; charter formulae; evidence; witness evidence; charter evidence; "Landeskundigkeit"; memory 


\section{Die Problemstellung}

In diesem Beitrag soll die Rolle der urkundlichen Dokumentation des Hochmittelalters nicht an der Produktion gemessen werden, sondern daran, wie diese Urkunden von den Zeitgenossen selbst verwendet worden sind. ${ }^{1}$ Schon bei der Untersuchung der hochmittelalterlichen Herrscherdiplome für das Patriarchat Aquileia ist dem Verfasser aufgefallen, dass diese Urkunden - soweit heute noch feststellbar - kaum anders verwendet worden sind, als dass spätere Herrscher bei Bestätigungen sich auf diese Vorgänger-Diplome berufen haben und dass sie diese auch als textliche Vorlagen benützten. ${ }^{2}$ Die Verwendung der Urkunde als prozessuales Beweismittel spielt demgegenüber eine sehr geringe Rolle. ${ }^{3}$

Der zweite Brünner Workshop zu diplomatischen Studien gab dem Verfasser Gelegenheit und Ansporn, sich neuerlich und eingehender als bisher mit der Frage zu befassen, welche Funktion(en) den Urkunden im Hochmittelalter zugedacht waren bzw. welche Rolle die Urkunden im Rechtsleben des Hochmittelalters tatsächlich gespielt haben. In Arengen, Publikationsformeln und Korroborationen betonen die Urkunden immer wieder, dass die schriftliche Fixierung das betreffende Rechtsgeschäft sichern solle. In welcher Weise diese Sicherung praktisch wirksam werden sollte, war für die Zeitgenossen offenbar so selbstverständlich, dass nähere Hinweise dazu in den Urkunden nicht eigens festgehalten werden mussten. Im Folgenden soll der Frage von deren praktischem Gebrauch nachgegangen werden, jetzt aber auf breiterer territorialer Grundlage und unter Einschluss der Privaturkunden. Zeitlich gilt die Untersuchung dem 11. und 12. Jahrhundert.

Angesichts der knappen verfügbaren Zeit musste sich der Verfasser auf die Dokumentation eines begrenzten Raums beschränken, konkret auf das Territorium der heutigen österreichischen Bundesländer Niederösterreich, ${ }^{4}$ Oberösterreich, ${ }^{5}$ Salzburg, ${ }^{6}$ Steier-

1 Infolge der umfangmäßigen Beschränkung der Beiträge für die Akten des zweiten Brünner Workshops zu diplomatischen Studien erscheint der vorliegende Beitrag hier in stark verkürzter Form. Um die Argumentationslinien möglichst intakt zu belassen, wurde insbesondere auf die in die Hunderte gehenden urkundlichen Einzelnachweise verzichtet; solche finden sich nur bei spezieller Anführung einzelner Stücke im Text. Die ungekürzte Fassung des Beitrags mit allen Einzel-Nachweisen erscheint im „Archiv für Diplomatik“. - Für wertvolle kritische Bemerkungen zum Entwurf dieses Beitrags sei Herrn Prof. Theo Kölzer auch an dieser Stelle der beste Dank ausgesprochen.

2 Herrscherurkunden für das Patriarchat Aquileia: Wann? Wozu? Vortrag am 6. Oktober 2011 in Magdeburg im Rahmen der Tagung „Herrscherurkunden für Empfänger in Lothringen, Oberitalien und Sachsen (9.-12. Jahrhundert)“. Die Tagungsakten sind noch nicht erschienen.

$3 \mathrm{Zu}$ diesen beiden Hauptverwendungen von Urkunden vgl. (mit Bezug auf die Karolingerzeit) Mersiowsky, Mark: Die Urkunde in der Karolingerzeit. Originale, Urkundenpraxis und politische Kommunikation II. Monumenta Germaniae Historica, Schriften 60-2. Wiesbaden 2015, S. 606, mit weiterer Literatur.

4 Niederösterreichisches Urkundenbuch (= NÖUB) I. Edd. M. Weltin - R. Zehetmayer; NÖUB II-1 und II-2. Edd. R. Zehetmayer - D. Weltin - M. Weltin. Publikationen des Österreichischen Instituts für Geschichtsforschung 8-1, 8-2/1 und 8-2/2. St. Pölten 2008 und 2013. Die von 1159 bis 1199 verbleibende Lücke wurde teilweise gefüllt durch das Urkundenbuch zur Geschichte der Babenberger in Österreich I. Edd. H. Fichtenau und E. Zöllner. Publikationen des Instituts für Österreichische Geschichtsforschung 3-1. Wien 1950.

$5 \quad$ Urkundenbuch des Landes ob der Enns (= OÖUB) II, Wien 1856.

6 Salzburger Urkundenbuch (= SUB) II. Edd. W. Hauthaler und F. Martin, Salzburg 1916. 
mark, ${ }^{7}$ Kärnten ${ }^{8}$ und Tirol (samt Teilen Südtirols), ${ }^{9}$ sowie auf das Gebiet der heutigen Republik Slowenien. ${ }^{10}$ Dazu kommen Ausblicke auf das Friaul ${ }^{11}$ und auf Istrien. ${ }^{12}$ Die in diesem Zusammenhang weniger ergiebigen Traditionsbücher wurden weniger vollständig erfasst als die „eigentlichen“ Urkunden. ${ }^{13}$ Auch unechte Urkunden waren einzubeziehen; schließlich sind sie besonders getreue Spiegel des jeweiligen Zeitgeistes. ${ }^{14}$ Sie sind zum guten Teil noch innerhalb des Untersuchungszeitraums entstanden bzw. sie stehen diesem überwiegend so nahe, dass sie noch als Spiegel der zeitgenössischen Auffassungen gelten können; die Aussagen, um die es hier geht, zeigen sich hier ganz offensichtlich als Fortsetzung einer hergebrachten Tradition und nicht als Neuerung ihrer eigenen Zeit. Im Übrigen ist damit zu rechnen, dass zumindest einige der für die hier vorliegende Studie relevanten Angaben in (späteren) unechten Urkunden aus echten Vorlagen stammen.

7 Urkundenbuch des Herzogthums Steiermark (= StUB) I, II und III. Ed. J. (v.) Zahn. Graz 1875,1879 und 1903. Neubearbeitung der (nach heutigen Begriffen) außersteirischen Provenienzen von Band I durch Friedrich Hausmann (= StUB H), 2007, online verfügbar unter der Adresse http://gams.uni-graz.at/ collection:stub.

8 Monumenta historica ducatus Carinthiae. Geschichtliche Denkmäler des Herzogtumes Kärnten (= MDC) I und III. Ed. A. v. Jaksch. Klagenfurt 1896 und 1904. Dazu I. Ergänzungsheft zu Band I-IV. Ed. A. v. Jaksch, Klagenfurt 1915 .

9 Tiroler Urkundenbuch II-1 und II-2. Edd. M. Bitschnau und H. Obermair. Innsbruck 2009 und 2012.

10 Urkunden- und Regestenbuch des Herzogtums Krain (= KrUB) I. Ed. F. Schumi. Laibach 1882/83. Hinsichtlich der Urkunden der Patriarchen von Aquileia ist dieses Werk überholt durch Documenta patriarchalia res gestas Slovenicas illustrantia / Listine oglejskih patriarhov za slovensko ozemlje in listine samostanov v Stični in Gornjem Gradu (1120-1251) / Patriarchenurkunden von Aquileia für Slowenien und die Urkunden der Klöster Sittich und Oberburg (1120-1251). Ed. G. Bernhard. Wien/Dunaj - Ljubljana 2006. Das untersteirische Gebiet ist im StUB mit erfasst; zu Marburg gibt es die Neuedition Gradivo za zgodovino Maribora I. Ed. J. Mlinarič. Maribor 1975.

11 In erster Linie sind hier relevant: Diplomi patriarcali. I documenti dei patriarchi Aquileiesi anteriori alla metà del XIII secolo nell'Archivio Capitolare di Udine. Ed. C. Scalon. Quaderni e dispense dell'Instituto di storia dell'Università degli Studi di Udine 8. Udine 1983; Die älteren Urkunden des Klosters Moggio (bis 1250). Ed. R. Härtel. Publikationen des Historischen Instituts beim Österreichischen Kulturinstitut in Rom 2-6-1. Wien 1985; Die älteren Urkunden des Klosters S. Maria zu Aquileia (1036-1250). Ed. R. Härtel. Publikationen des Historischen Instituts beim Österreichischen Kulturforum in Rom 2-6-2. Wien 2005.

12 In erster Linie ist hier relevant: Codice diplomatico istriano. Ed. P. Kandler. Loseblattausgabe ohne Paginierung und Nummerierung. Fünfbändiger Neudruck mit Paginierung und Nummerierung: Trieste 1986.

13 Berücksichtigt wurden: alle Salzburger Traditionsbücher in SUB I. Ed. W. Hauthaler. Salzburg 1910; Die Traditionsbücher des Benediktinerstiftes Göttweig. Ed. A. F. Fuchs. Fontes rerum Austriacarum II-69. Wien Leipzig 1931; Die Traditionsurkunden des Klosters Garsten. Kritische Edition. Ed. S. Haider. Quelleneditionen des Instituts für Österreichische Geschichtsforschung $8=$ Forschungen zur Geschichte Oberösterreichs 20. Wien - München 2011. Die weiteren Traditionsbücher aus dem hier untersuchten Raum wurden insoweit berücksichtigt, als deren Einträge in den weiter oben angeführten Urkundenbüchern enthalten sind. Auf diese Weise sind zugleich die Traditionsbücher von Admont und von St. Paul vollständig erfasst.

14 Vgl. Constable, Giles: Forgery and Plagiarism in the Middle Ages. Archiv für Diplomatik 29, 1983, S. 1-41, hier 2. 


\section{Bestätigungen von Urkunden}

Vorauszuschicken ist, dass jenes Bild, welches der Verfasser aus den Herrscherdiplomen für Aquileia gewonnen hat, mit anderen Untersuchungen ähnlichen Zuschnitts übereinstimmt: In der auf uns gekommenen Überlieferung werden solche Urkunden viel häufiger schlicht bestätigt als vor Gericht präsentiert. ${ }^{15}$ Für Herrscher stellen Bestätigungen einen wichtigen Teil ihrer Regierungshandlungen dar, ${ }^{16}$ und damit auch ihrer Beurkundungen. ${ }^{17}$ Dasselbe gilt für die Privaturkunden, ${ }^{18}$ und das auch in dem hier untersuchten Raum. Wo Konkretes zum Urkundengebrauch zu erfahren ist, bestätigt in den meisten Fällen ein Rechtsnachfolger die Urkunde eines Amtsvorgängers oder eine übergeordnete Instanz die Urkunde einer untergeordneten. Dazu kommt als Sonderfall jene Korrespondenz mit der römischen Kurie, mit der von mehreren Seiten her die Bestätigung von Garstener Privilegien erbeten wurde. Freilich ist nicht von vornherein klar, ob der Hinweis auf zur Bestätigung vorgelegte Urkunden nicht auch als formelhaft angesehen werden kann. ${ }^{19}$ Aber auch eine solche Formel muss ihrerseits aus einem konkreten Gebrauch entstanden sein, und damit ist sie zumindest als Spiegel einer einstigen "Normalität" anzusehen.

Natürlich bedeutet das noch nicht, dass Urkunden von vornherein zum Zweck späterer Bestätigung ausgefertigt worden sind, und ebenso wenig, dass die Vorlage einer Urkunde zwecks Bestätigung der häufigste Fall von hochmittelalterlichem Urkundengebrauch gewesen sein muss. Schließlich kamen als bestätigende Instanzen fast nur Herrscher, Päpste, Herzöge und Bischöfe in Frage. Aber zumindest für die Herrscherurkunden kann als gesichert gelten, dass das Bestätigen nicht nur von juristischer, sondern ebenso auch von politischer Bedeutung war, und das nicht nur für die Empfänger, ${ }^{20}$ sondern auch für die Aussteller ${ }^{21}$. Bestätigungsketten wurden als besondere Gewähr der Rechtssicherheit betrachtet. ${ }^{22}$

15 Krause, Hermann: Die Rolle der Bestätigung in der Hohenstaufenzeit. Dargelegt an den frühen Barbarossa-Urkunden. In: Rechtsgeschichte und Rechtsdogmatik. Festschrift Hermann Eichler zum 70. Geburtstag am 10. Oktober 1977. Hg. von U. Flossmann. Linzer Universitätsschriften. Festschriften 1. Wien - New York 1977, S. 387-409. Zur älteren Zeit vgl. Mersiowsky, M.: Urkunde II, S. 749.

16 Zu Bestätigungen allgemein ebd., S. 624-646 und 749-766.

17 So (mit Bezug auf die Karolingerzeit) ebd., S. 624; vgl. ebd., S. 632 zu Ludwigs des Frommen systematischer (und auch in den Arengen dokumentierter) Bestätigungspolitik.

18 So mit Bezug auf die Karolingerzeit ebd., S. 762 und 876-882; zur Privaturkunde als Beweismittel vgl. ebd., S. 891-897.

19 Vgl. (mit Bezug auf die Karolingerzeit) ebd., S. 606.

20 So (mit Bezug auf die Karolingerzeit) ebd., S. 638, 646 und 765.

21 So ebd., S. 646 und 763.

22 Dazu ebd., S. 758-759 und 763-764. 


\section{Urkunden als Beweismittel}

Demgegenüber macht der Urkundengebrauch für Beweiszwecke in Streitsachen ${ }^{23}$ zahlenmäßig nur einen Bruchteil der Bestätigungen aus, und zwar auch bei großzügigster Berechnung nur etwa ein Sechstel. ${ }^{24}$ Und auch hiervon sind italienische Gerichtsurkunden, ${ }^{25}$ bloß in Aussicht genommene eventuelle Verwendung, ${ }^{26}$ bloß behaupteter Urkundenbesitz bzw. das bloße Anerbieten der Vorlage von Urkunden, allgemein bekannte Dekrete und verkappte Bestätigungen ${ }^{27}$ nicht voll $\mathrm{zu}$ werten. Es fällt auch schwer anzunehmen, dass sich Beweis und Bestätigung auf die Überlieferungschance von Urkunden unterscheidlich ausgewirkt hätten. Es scheint tatsächlich, als ob der Beweiszweck der Urkunden bislang eher zu hoch eingeschätzt worden ist. ${ }^{28}$

Gelegentlich wird berichtet, dass sich ein Widersacher durch eine Urkunde überzeugen ließ und von seinen Ansprüchen Abstand nahm. Das liegt gewissermaßen an der Grenze zum gerichtlichen Beweis. Ähnlich steht es um Urkunden-Erneuerungen anlässlich aktueller Anfechtungen. Nach alledem verbleiben nur mehr sehr wenige wirklich zuverlässige Belege für die Verwendung von Urkunden als Beweismittel vor Gericht, und mehrere davon sind für die Urkunde nicht einmal schmeichelhaft. Einmal wurde eine Urkunde als Fälschung verworfen und die Gegenpartei obsiegte durch Zeugenbeweis. Wo wir die Urkunde vereinzelt als turris fortitudinis a facie inimici et persequentis hingestellt finden, ${ }^{29}$ dort verweist das wohl eher auf Wunschdenken als auf Realität. Es verdiente nähere Untersuchung, in welchem Ausmaß Fälschungen dazu bestimmt waren, als

23 Von den seltenen (bzw. selten überlieferten) Gottesurteilen ist hier abzusehen. Ein gerichtlicher Zweikampf findet sich in Istrien, eine Abschaffung der Wasserprobe in Niederösterreich. Zur geringen Zahl der Gottesurteile vgl. Brunner, Walter: „... damit alle dem Urteil Gottes ohne Zweifel glauben!“ Gottesurteile als magisches Mittel der Wahrheitsfindung im Südostalpenraum. In: Blätter für Heimatkunde [Steiermark] 80, 2006, S. 68-78. Hier werden auch nichturkundliche Quellen (und weit über 1200 hinaus) berücksichtigt. Brunner sieht die geringe Zahl an Nachrichten vorrangig als Folge der archivalischen Überlieferung (ebd., S. 73).

24 Zum Urkundenbeweis im fraglichen Raum und zur selben Zeit vgl. bereits Härtel, Reinhard: Schrift und Gericht. In: Schriftkultur zwischen Donau und Adria bis zum 13. Jahrhundert. Akten der Akademie Friesach „Stadt und Kultur im Mittelalter“ Friesach (Kärnten), 11.-15. September 2002. Hg. von R. Härtel u. a., red. von Ch. Domenig. Schriftenreihe der Akademie Friesach 8. Klagenfurt 2008, S. 363-397, hier 388-389.

25 Diese Stücke entstammen einer deutlich anderen Dokumentationskultur, am umfassendsten dokumentiert in: I placiti del „Regnum Italiae“ II. Ed. C. Manaresi. Fonti per la storia d'Italia 96. Roma 1957. Zur Urkunde als Beweismittel in italienischen Gerichtsurkunden bereits der Karolingerzeit vgl. zuletzt Mersiowsky, M.: Urkunde II, S. 733. Auch der schriftliche Niederschlag des voll entwickelten römisch-kanonischen Prozessverfahrens erscheint innerhalb des Untersuchungsraums zuerst im Süden

26 Einmal findet sich (in einer Sicherungsformel) auch die Bestimmung, dass nur Privilegien von ganz bestimmter Art als Gegenbeweis zugelassen sein sollten.

27 Wenn ein Bischof nach Streitigkeiten mit seinem Domkapitel die Privilegien seiner Vorgänger schließlich anerkannte, dann steht dieser Vorgang einer Bestätigung näher als einem Urkundenbeweis. Erst recht gilt das für urkundlich „bewiesene“ Rechte, die einer eindeutigen Bestätigung zugrunde liegen.

28 Vgl. Mersiowsky, M.: Urkunde II, S. 605: „Zu sehr wurde die Urkunde als Beweismittel und bloßer Text gesehen, als dass man sich mit der Vorlage älterer Dokumente näher beschäftigte“.

29 OÖUB II, S. 275-276, Nr. 185. Genau dieselben Worte werden wenig später für einen potenten Beschützer gebraucht: StUB H, Nr. Wi 6 (= OÖUB II, S. 313-314, Nr. 213). 
Grundlage einer Bestätigung Rechte abzusichern oder zu erlangen, und in welchem Umfang sie von vornherein als Beweismittel in gerichtlichen Verfahren dienen sollten.

Wo wir Näheres über die Beweismittel erfahren, regierten die Zeugen, ${ }^{30}$ von denen allerdings oft nicht hinreichend deutlich die Rede ist. Dann und wann erscheinen die Zeugen zusammen mit Urkunden, ohne dass ein Vorrang des einen oder des anderen Beweismittels eindeutig erkennbar wäre. Gelegentlich wurde ausdrücklich die Erwartung geäußert, neben den lebendigen Zeugen möge auch die gesiegelte Urkunde als Zeuge dienen. Meistens jedoch finden sich Zeugen allein, ohne urkundliche „Konkurrenz", und das (ebenso wie Schwurleute) sogar in italienischen Gerichtsurkunden. Nur Einzelheiten erfahren wir so gut wie nie. Denn im Hochmittelalter war bei der schriftlichen Fixierung gerichtlicher Handlungen die Möglichkeit zum Nachvollzug eines bereits durchgeführten Verfahrens normalerweise nicht der Hauptzweck; dazu kam es erst im ausgehenden 13. Jahrhundert infolge der Verbreitung des römisch-kanonischen Prozess-

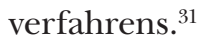

Die Arengen preisen bekanntlich immer wieder die schriftliche Fixierung als Mittel, die Erinnerung an eine Rechtshandlung wachzuhalten. Diesen Zweck hätten allerdings auch schlichte Erzählungen erfüllt, ohne Einbettung in einen urkundlichen Text. Und auch mit der Beweisfunktion der Urkunde (speziell der Privaturkunde) war es, soweit die Überlieferung erkennen lässt, noch nicht weit her. Eine Beweisfunktion der Urkunde sprechen die Arengen auch niemals an, oder nur so verschwommen, dass auch der Zweck bloßer Information gemeint sein kann. Wofür waren dann all die vielen Urkunden gedacht? Gibt es eine Art Brücke zwischen der vielbeschworenen urkundlichen Erinnerung und den wenigen urkundlichen Beweisführungen? Eine solche Brücke könnte sein, dass die Urkunden nicht unbedingt etwas „beweisen“ sollten. Es gibt etliche Zeugnisse dafür, dass man in den Urkunden etwas „finden“ oder aus ihnen etwas „erfahren“ bzw. „erkennen“ konnte, und dass die Niederschrift von Rechtshandlungen auch von vornherein gerade diesen Zweck verfolgt hat. Auf dieses urkundlich gestützte Wissen wird bald zurückzukommen sein.

\section{Urkunden als Exempla}

Zuvor ist aber noch zu fragen, ob oder inwieweit die Zeugnisse für den Urkundengebrauch - häufig zur Bestätigung, selten zum Beweis - ein getreues Abbild der einstigen Wirklichkeit liefern. Hierbei ist zunächst festzuhalten, dass neuere Erkenntnisse über

30 Zum Zeugenbeweis im fraglichen Raum und zur selben Zeit vgl. bereits Härtel, R.: Schrift und Gericht, S. 386-388. Grundlegend Hageneder, Othmar: Die geistliche Gerichtsbarkeit in Ober- und Niederösterreich. Von den Anfängen bis zum Beginn des 15. Jahrhunderts. Forschungen zur Geschichte Oberösterreichs 10. Linz 1967, hier besonders S. 114-115 zur Zulassung auch (!) von Urkunden neben den Zeugenaussagen (frühes 13. Jh.). Zum Erzbistum Salzburg vgl. Paarhammer, Johann: Die geistliche Gerichtsbarkeit. In: Geschichte Salzburgs. Stadt und Land I-2. Hg. von H. Dopsch. Salzburg 1983, S. 1054-1070, hier 1055.

31 Vgl. Härtel, R.: Schrift und Gericht, S. 386. Zur namentlichen Nennung von befragten Zeugen vgl. ebd., S. 386-387. Die Protokollierung einzelner Zeugenaussagen nach dem Muster NN iuratus dixit ist eine Spezialität des Südens und kommt auch dort erst kurz vor 1200 vor; vgl. ebd., S. 387-388. 
früh- und hochmittelalterliche Gerichtsverfahren diese weithin als Plattformen sehen, auf denen mit gesellschaftlichem Druck die Streitparteien zu einer Einigung gedrängt werden sollten. Diese Sichtweise entspricht vorzüglich dem Befund, dass von sehr vielen Gerichtsverfahren ein förmlicher Abschluss nicht bekannt ist, bzw. dass dieser Abschluss nur allzuoft in einer „Transaktion“ bestand. ${ }^{32}$ Es ist offensichtlich, dass in einer solchen Welt der Urkundenbeweis nicht sehr viel Gewicht haben konnte.

Die urkundlichen Sicherungsformeln, daneben auch die Pönformeln und die Korroborationen sind geeignet, diesen Eindruck massiv zu unterstützen. In diesen Formeln versuchen die Aussteller, die Nachwelt zur Beobachtung der soeben beurkundeten Verfügungen zu veranlassen. Den Standardfall bilden jene Formulierungen, in denen sich der Aussteller an die Nachwelt insgesamt wendet. Nicht selten aber wendet er sich in erster Linie an seine eigenen Rechtsnachfolger. Noch viel häufiger wendet sich der Aussteller an seine Rechtsnachfolger allein. Das gilt vor allem für die Bischöfe und für Aussteller geringeren Ranges. Immer wieder ermahnen die Aussteller ihre Rechtsnachfolger auch nur, oder sie bitten sie flehentlich, den beurkundeten Rechtsinhalt nicht anzutasten, oder sie wollen ihnen nur Vorbild sein. Auch tatsächlich erfolgte Übertretungen durch Rechtsnachfolger werden angesprochen.

Es sieht alles danach aus, als hätten die Aussteller ihre eigenen Rechtsnachfolger als Hauptgefahr für den dauernden Bestand der beurkundeten Rechtshandlung angesehen, ${ }^{33}$ und sehr wahrscheinlich war das auch die Sichtweise der Urkundenempfänger. ${ }^{34}$ Die in der Überlieferung vorhandene Fülle urkundlicher Bestätigungen mag (auch) von daher gesehen verständlich erscheinen.

Das alles fügt sich gut zu dem eingangs angesprochenen Exemplum-Charakter, wie er Urkunden von bestätigenden Rechtsnachfolgern, formelhaft oder nicht, häufig zugesprochen wird. Auch in den Privaturkunden wurde man nicht müde, den Ausstellern von Bestätigungsurkunden das gute Vorbild von deren Vorfahren bzw. Amtsvorgängern in den Mund zu legen.

Die Frage, wie verbindlich oder wie unverbindlich ein solches exemplum für den Rechtsnachfolger war, wurde im Schrifttum schon mehrfach - und widersprüchlich erörtert. ${ }^{35}$ Ebenso widersprüchlich sind die urkundlichen Aussagen aus dem Untersuchungsraum. Sie reichen von angedeuteten Nützlichkeits-Vorbehalten bis zur bloßen Ermahnung an die Adresse des Nachfolgers. Die in diesem Zusammenhang verwendeten Verben reichen von debet bis decet und zeigen damit auch ihrerseits an, dass es sich hier

32 Ebd., S. 374-381 und 396-397, mit weiterer Literatur.

33 Das zeigt sich auch, wenn der wesentliche Zweck einer urkundlichen Niederschrift (von 1190) darin gesehen wird, dass bei den Rechtsnachfolgern des Ausstellers und dessen Verwandtschaft kein Zweifel über das Rechtsgeschäft bestehen soll.

34 Wo ein Kloster mit dem Rechtsnachfolger eines Wohltäters im Streit lag und zum Mittel der Urkundenfälschung griff, dort lag eine Verbotsbestimmung gegen die Nachfolger des einstigen Gönners natürlich besonders nahe.

35 Dazu Michael Borgolte: „Ob eine Verleihung, ein Privileg, über die Lebensdauer des Verleihenden und auch des Beliehenen hinaus Kraft haben würde, war letztlich abhängig von der Duldung oder Bestätigung durch die Nachfolger (so nach Mersiowsky, M.: Urkunde II, S. 750). Beobachtungen zu Usus einerseits und Verpflichtung andererseits (mit Bezug auf die Karolingerzeit) bei Mersiowsky, M.: Urkunde II S. 751-753. 
um eine Grauzone zwischen Recht und Anstand handelt. Dem entsprechen einzelne ganz unmissverständliche Nachrichten, dass Rechtsnachfolger die Inhalte der ihnen vorgelegten Vorgänger-Urkunden vor der Bestätigung auf ihre aktuelle Nützlichkeit und Angemessenheit überprüften. Und es musste auch keineswegs immer der gesamte Inhalt vorgelegter Urkunden Bestätigung finden.

Die Rechtsnachfolger eines Ausstellers waren an dessen Verfügungen also nicht strikt gebunden. Umso eher konnten sie als die größte Gefahr für den dauernden Bestand der beurkundeten Handlung angesehen werden, und umso eher mochten es Empfänger als zentral angesehen haben, sich - so wie bei Herrschern ${ }^{36}$ - auch bei bischöflichen oder herzoglichen Rechtsnachfolgern um eine Bestätigungsurkunde zu bemühen und diese auf die Wahrung des aktuellen Rechtszustandes zu verpflichten. Und es ist klar, dass zur Erlangung dieses Zwecks - unter gewöhnlichen Umständen - ein Aufmarsch von Zeugen weit weniger tunlich war als eine beispielgebende Urkunde des Amtsvorgängers selbst, am besten mit dessen Siegel. Hier vor allem war das Feld, auf dem die Urkunde ihre Wirkung entfalten konnte, viel eher als vor Gericht.

Nach alledem wird es nicht zu gewagt sein, die Urkundenvorlage zwecks Bestätigung tatsächlich für den Normalfall des hochmittelalterlichen Urkundengebrauchs in unseren Breiten zu halten, zumindest im Fall höherrangiger Aussteller.

\section{Erneuerte Zeugen}

Über die rechtlichen Aspekte der wiederholten Bestätigungen ist schon intensiv nachgedacht worden. ${ }^{37}$ Es lässt sich auch ein praktischer Aspekt namhaft machen, und dieser führt auf die Rolle der Zeugen zurück. Die fortgesetzten Bestätigungen mochten neben der Erneuerung von Urkunden auch dazu gedacht gewesen sein, in feierlichen Akten mit vornehmer Zeugenschaft - und womöglich in Gegenwart potentieller Aggressoren - den einmal beurkundeten Rechtszustand immer wieder in Erinnerung zu rufen. Das musste es potentiellen Angreifern erschweren, gegen die solcherart gestärkten Rechte zu verstoßen. Dazu kam die - wohl ebenfalls beabsichtigte - Folge, dass mit jeder Erneuerung immer wieder „frische“ Zeugen zur Verfügung standen, während die ursprünglichen Handlungszeugen längst verstorben waren.

Fallweise tritt eine solche Denkweise sogar offen zutage. Eine St. Pauler Urkunde von 1124 berichtet, dass die Zeugen einer Güterübergabe verstorben waren. Aber der Abt konnte den noch lebenden Schenker dazu bewegen, seine frühere Schenkung innovatis testibus zu bestätigen. ${ }^{38}$ Fortgesetzte Bestätigungen konnten also Erneuerung der Erinnerung mit erneuerter Zeugenschaft bedeuten. Andererseits ist klar, dass diese Deutung nicht überall greift, so etwa bei fortgesetzten päpstlichen Schutzprivilegien. Aus deren

36 Dorn, Franz: Die Landschenkungen der fränkischen Könige. Rechtsinhalt und Geltungsdauer. Rechts- und Staatswissenschaftliche Veröffentlichungen der Görres-Gesellschaft. N.F. 60. Paderborn u. a. 1991.

37 So bereits Krause, Hermann: Dauer und Vergänglichkeit im mittelalterlichen Recht. Zeitschrift für Rechtsgeschichte. Germanistische Abteilung 75, 1958, S. 206-251. 
Kardinals-Unterfertigungen waren ganz gewiss keine neuen Zeugen zu gewinnen, die man vor Gericht präsentieren konnte.

\section{Die Rolle der Landeskundigkeit}

Es stellt sich die Frage, wie sich eine solche Sicht fortgesetzter Absicherung mit der Anschauung von der allgemeinen Landeskundigkeit als Sicherung vor Anfechtungen und Übergriffen verträgt. Die traditionelle Auffassung ist, dass Notitien die Zeugen festhielten und dass nach deren Tod Besitznachbarn an deren Stelle traten. Dazu kommt die Öffentlichkeit oder Offenkundigkeit bzw. die Landeskundigkeit der Handlung. ${ }^{39}$ In jüngster Zeit hat Roman Zehetmayer dazu den neuen Akzent gesetzt, dass nach dem Tod der Handlungszeugen der Rechtszustand durch Ersitzung gesichert war. ${ }^{40}$

Ganz gewiss haben alle diese Elemente ihre Bedeutung. Aber sie fügen sich in dieser Weise doch noch nicht wirklich zusammen. Wenn lange Zeit nach dem Rechtsakt ein Übeltäter fremdes Gut mit Gewalt dessen rechtmäßigem Besitzer entzog, wie konnte der Geschädigte dann die Landeskundigkeit für sich nutzbar machen? Nach Laut der Publikationsformeln sollte zumeist die gesamte Christenheit die Rechtslage kennen, aber wie sollte diese Allgemeinheit dem Geschädigten helfen? In einem förmlichen Verfahren kam es ja doch wieder nur auf einen engeren Kreis zuverlässiger und sachkundiger Personen an: entweder Zeugen der rechtlich grundlegenden Handlung oder wenigstens Zeugen der Sachlage. Niemals ist die Rede davon, dass ein Übergriff mit Hinweis auf die allgemein bekannte Rechtslage verurteilt worden wäre.

Die Landeskundigkeit wird normalerweise, wenn auch nur indirekt, in der Publikationsformel angesprochen. Allerdings wird deutlich, dass in diesen Formeln insgesamt weniger die Publikation (als Handlung), sondern in erster Linie die Publizität (und damit das Ergebnis) im Vordergrund steht. In jedem Fall aber zielen diese Formeln von allem Anfang an auf die allgemeine Bekanntheit des Rechtsgeschäfts und vielfach auch auf den Schutz vor dem Vergessenwerden.

Es liegt somit nahe anzunehmen, dass die Bedeutung der Landeskundigkeit nicht so sehr in einer Art Zeugen-Ersatz erst nach deren Tod gelegen war, und es ist möglich, einen doppelten „Schutzwall“ für das Rechtsgeschäft zu sehen: Einen äußeren Ring bildete die allgemeine Bekanntheit, die es einem potentiellen Rechtsverletzer von vornherein erschweren musste, ein Rechtsgeschäft anzufechten oder sich mit Gewalt über dieses hinwegzusetzen. Wenn man einer Zeugenformel aus Herzogenburg (aus dem Jahre 1150) trauen will, dann reichte für diese allgemeine Bekanntheit die bloße fama. ${ }^{41}$

39 So Zehetmayer, Roman: Urkunde und Adel. Ein Beitrag zur Geschichte der Schriftlichkeit im Südosten des Reichs vom 11. bis zum frühen 14. Jahrhundert. Veröffentlichungen des Instituts für Österreichische Geschichtsforschung 53. Wien - München 2010, S. 55-56 und 62, mit vorangehender Literatur. Zur Diskussion um die Bedeutung der öffentlichen Verlautbarung als der höchsten Gewähr für die Rechtssicherheit vgl. Zehetmayer, R.: Urkunde und Adel, S. 62.

40 Ebd., S. 83-84.

41 NÖUB II-1, S. 385-390, Nr. 10/5. 
Jedenfalls weisen die Publikationsformeln bisweilen auch auf das Bewusstsein hin, dass der angestrebte weite Kreis der „Wissenden“ seine Kenntnis des Rechtsgeschäfts nur aus dem Hörensagen haben konnte; ${ }^{42}$ mehrfach wird klar die Erwartung ausgesprochen, dass die Rechtshandlung von Generation zu Generation weitererzählt werden solle. Der innere Sicherungsring wurde dann von den Zeugen gebildet. Auf diese musste man zurückgreifen, wenn - trotz allgemeiner Bekanntheit - infolge Anfechtung oder gewalttätigen Übergriffs ein förmliches Verfahren unvermeidlich wurde. ${ }^{43}$ Verhielt es sich so, dann hat zunächst eine allgemeine Memoria den Rechtszustand garantiert, notfalls aber musste die spezielle Memoria der Zeugen als Beweismittel fungieren. Pointiert ausgedrückt: Die Landeskundigkeit war mehr ein Schutz gegen die Vergesslichen, aber gegen Böswillige halfen nur Zeugen.

Wenn dieses Szenario zutrifft: Welche Rolle spielte in ihm die Urkunde? Es wird nicht allzu verwegen sein, die Traditionsnotizen - und sogar die frühen Siegelurkunden - nicht zuletzt als Gedächtnisstützen für Zeugenaussagen anzusehen. Eine solche Rolle von urkundlichen Niederschriften ist für die Schöffengerichte im nordfranzösisch-rheinischen Raum seit langem nachgewiesen. ${ }^{44}$

\section{Urkunden als Grundlage für Zeugenaussagen}

Es scheint, dass sich diese Sichtweise durch die weit verbreiteten Vergessensarengen stützen lässt, in denen die rechtssichernde Rolle der Schrift hervorgehoben wird. Nimmt man diese Arengen (und inhaltlich verwandte Formel-Aussagen) wörtlich, so beruhte die Sicherheit des Rechtsgeschäfts nicht so sehr auf der Niederschrift als solcher, sondern es war die Funktion der Schrift, in der so vergesslichen Welt die Erinnerung wachzuhalten oder jedenfalls zu informieren. Es ging nicht darum, im strengen Sinne des Wortes zu „beweisen“.

Wenn dem so war, dann mochte man für die Abwehr von Anfechtungen oder Übergriffen auch bei der Niederschrift von Urkunden nicht nur an deren unmittelbare Vorlage, sondern mindestens ebenso sehr an mündliche Aussagen (nämlich aufgrund der

42 Dies gilt für Publikationsformeln mit dem Beginn Audiant bzw. Audiat. Dasselbe gilt für die Aussage, dass durch das Hören Gewissheit erlangt werden könne.

$43 \mathrm{Zu}$ der bisherigen Ansicht, dass ein über die eigentlichen Handlungszeugen hinausgehender Kreis für die Rechtsfindung herangezogen werden konnte, vgl. Zehetmayer, R.: Urkunde und Adel, S. 62. Bei Zehetmayer ist der Zeugenbeweis „sehr mit Mängeln behaftet, die auch von der Offenkundigkeit nicht gänzlich beseitigt werden konnten“, so ebd., S. 64. In der Sichtweise des Verfassers würde der Zeugenbeweis erst dort und dann zum Zug gekommen sein, wo die Landeskundigkeit als Schutzwall nicht mehr ausreichte.

44 de Boüard, Alain: L'acte privé. Manuel de diplomatique française et pontificale II. Paris 1948, S. 233, 235 und 247; Militzer, Klaus: Schreinseintragungen und Notariatsinstrumente in Köln. In: Notariado público y documento privado: de los orígenes al siglo XIV. Actas del VII Congreso Internacional de Diplomatica, Valencia, 1986. Papers i Documents 7-2. Valencia 1989, S. 1195-1224, hier 1201-1202. Zur Urkunde als Unterstützung des Gedächtnisses auch Guyotjeannin, Olivier: „Penuria scriptorum”. Le mythe de l'anarchie documentaire dans la France du nord ( $X^{e}$ - première moitiè du XI siècle). In: Pratiques de l'écrit documentaire au XI ${ }^{\mathrm{e}}$ siècle. Hg. von O. Guyotjeannin - L. Morelle - M. Parisse. Bibliothèque de l'Ècole des chartes 155, 1997, S. 11-44, hier 13-14. 
Niederschrift) gedacht haben. Auch das würde sich gut zum urkundlich feststellbaren Verwendungsbild fügen: Bestätigungen häufig, (unmittelbare) Verwendung vor Gericht selten. Dies könnte zugleich eine Antwort auf die Frage sein, ob nämlich den Traditionsbüchern wirklich solche Beweiskraft zukam und ob die Traditionsnotizen vollgültige Urkunden waren, wie dies eben jüngst noch Joachim Wild gesehen hat, ${ }^{45}$ und das wohlgemerkt - nicht nur in den Angelegenheiten von Kloster-Hörigen ${ }^{46}$ Für eine solche Beweiskraft gibt es auch aus dem hier untersuchten Raum keinen Beleg. Konnten Traditionsbücher jedoch für das „Briefing“ von Zeugen verwendet werden, dann erfüllten sie eben auf indirekte Weise diesen Zweck, ohne dass man die wertvollen Schriften all den Gefahren aussetzen musste, wie sie Reisen notwendigerweise mit sich brachten. Und wenn man das mit Traditionseinträgen so halten konnte, dann wohl auch mit förmlicheren Urkunden.

Es hat freilich etwas Unbefriedigendes an sich, solche Schlüsse über rechtliche Praktiken im Wesentlichen aus einem Arengentyp (der Vergessensarenga) abzuleiten. Aber es gibt in den Urkunden auch konkretere Aussagen zu diesem Thema, wenn auch nur fallweise. 1145 bezeichnete Bischof Reginbert von Passau eine von ihm ausgestellte Urkunde als certissimum memoriale für Gegenwärtige und Zukünftige ${ }^{47}$ So ein memoriale scheint einer Gedächtnisstütze zum auch forensischen Gebrauch deutlich näher zu stehen als einem unmittelbaren Beweismittel. Deutlicher noch ist die Erwartung, die künftige mündliche Überlieferung einer Rechtshandlung möge im Sinne der vorliegenden Niederschrift erfolgen: Unde presentis stili assertione filii qui nascentur et exsurgent narrent filiis suis qualiter..${ }^{48}$ Und auch wenn man das alles noch für eher schwache Zeugnisse halten sollte, so spricht eine Gurker Urkunde vom Ende des 12. Jahrhunderts wirklichen Klartext. Diese fordert nämlich ihre künftigen Leser unmissverständlich auf, nach Kenntnisnahme des Inhalts als geeignete Zeugen zur Verfügung zu stehen: Quicumque autem ista legerit, rogamus, ut huius rei, sicut a literis istis accepit, testis idoneus inveniatur. ${ }^{49}$ In der Vorstellung dieses Urkundenredaktors genügte es, dass der Zeuge seine Kenntnis aus einer Urkunde bezogen hatte.

Nun gibt es zweierlei Möglichkeiten, vereinzelte und auffällige Ausdrucksformen zu beurteilen: Man kann sie als Ausnahmeerscheinungen abtun oder aber als glückliche

45 Wild, Joachim: Wurzeln und Entwicklungslinien der privaten Siegelurkunde in Bayern (ca. 1150-1250), in: Mitteilungen des Instituts für Österreichische Geschichtsforschung 116, 2008, S. 235-248.

46 Der Verfasser gehört zu denjenigen, die der von Heinrich Wanderwitz und seitdem auch noch von Joachim Wild vertretenen Ansicht von der öffentlichen Glaubwürdigkeit von Traditionsbüchern nicht folgen können. Die für diese Ansicht bisher angeführten „Belegstellen“ beziehen sich auf Kloster-Hintersassen oder sind die offensichtliche Folge von Wunschdenken, und Urkundenkopien am Beginn von Traditionsbüchern enthalten nicht mehr rechtlichen Anspruch als vorangestellte Fundationsberichte; vgl. dazu Zehetmayer, R.: Urkunde und Adel, S. 64-69. Auch päpstliche Bestätigungen sind - anders als schon vermeint - wohl nicht aufgrund vorgelegter Notitien ausgefertigt worden, vgl. ebd., S. 74. Es gibt keinen Beleg dafür, dass (unbesiegelte) Notitien oder Traditionsbücher vor Gericht als Beweismittel vorgelegt worden wären, vgl. ebd., S. 76. Dem widerspricht nicht, dass Petenten mit derartigen Schriften fallweise Eindruck machen konnten.

47 NÖUB II-1, S. 183-185, Nr. 5/8 (= SUB II, S. 337-338, Nr. 235).

48 MDC III, S. 530-531, Nr. 1387 (= StUB II, S. 19-21, Nr. 4).

49 MDC I, S. 238-239, Nr. 317. 
Einzelfälle würdigen, in denen sich solches Gedankengut der Zeit manifestiert, das wegen seiner Selbstverständlichkeit normalerweise nicht eigens mitgeteilt wird. Der Verfasser neigt dazu, insbesondere die Aussage der Gurker Urkunde als eine Art Fenster in eine sonst unsichtbar gewordene Vergangenheit zu würdigen, auch wenn es zumindest bislang keine weiteren Beweise von gleicher Eindeutigkeit für eine solche Zwecksetzung der Urkunde gibt. Für das hier ganz eindeutig angesprochene urkundliche „Briefing“ von Zeugen sprechen allerdings auch jene umfänglichen urkundlichen Güterverzeichnisse, bei denen es von vornherein ausgeschlossen scheint, dass sich ein Mensch das alles über viele Jahre hinweg merken konnte, und zu denen am Schluss dennoch Zeugen angeführt werden. ${ }^{50}$

Dass „Zeugen aus zweiter Hand“ im Hochmittelalter Realität gewesen sind, zeigt sich auch dort, wo Zeugenaussagen protokolliert worden sind. Die Zeugnisse dieser Art sind allerdings nur sehr wenige, und diese stammen alle aus dem Friaul bzw. aus Istrien, und zwar aus den letzten Jahren des Untersuchungszeitraums bzw. unmittelbar danach. Hier kommen auch Zeugen zu Wort, welche ihr Wissen nur vom Hörensagen hatten; allerdings wird hier jeweils festgehalten, ob der betreffende Zeuge dies oder jenes selbst erlebt, gesehen oder (nur) gehört hatte. Gelegentlich beruhte eine Zeugenaussage erklärtermaßen auf einer Urkunde, deren Inhalt dem Zeugen nur durch Vorlesen bekannt geworden war. Auch hier darf vermutet werden, dass ein solcher Vorgang zwar nur ausnahmsweise dokumentiert erscheint, in der einstigen Wirklichkeit aber zur Normalität gehört haben mag.

Was ergibt sich aus alledem? Natürlich mochte eine Urkunde im Einzelfall auch selbst als „Zeuge“ gegolten haben. Trotzdem können urkundliche Niederschriften in vielen Fällen - gleich ob von Anfang an so beabsichtigt oder nicht - ein Mittel gewesen sein, um entsprechende Zeugenaussagen sicherzustellen. Dass Zeugen wohl versichern konnten, etwas gesehen oder gehört zu haben, niemals aber sich auf Gelesenes beriefen, steht dem nicht unbedingt entgegen. Denn die Verlesung von Urkunden gehörte zur Normalität. Weltliche Zeugen wären von vornherein kaum je in der Lage gewesen, eine Urkunde selbst zu lesen und zu verstehen. Was ein Zeuge gehört hatte, konnte also auch der Text, die Übersetzung oder die Erläuterung einer Urkunde gewesen sein. Die Urkunde konnte ihm auch gezeigt worden sein und insbesondere das Siegel des Ausstellers ihn beeindruckt haben. Von dieser Seite her betrachtet, muss die unleugbare Zunahme der Urkundenproduktion nicht unbedingt einer wachsenden Bedeutung des (noch eher unbedeutenden) Urkundenbeweises entsprochen haben. Noch im Jahre 1197 erklärte ein Millstätter Mönch als Redaktor einer Urkunde Erzbischof Adalberts von Salzburg, womit man in dieser veränderlichen Welt Rechtsakte absichern könne, nämlich privilegiis et maxime pluribus testibus. ${ }^{51}$ Es scheint, dass sich hier, anstelle all der eher stereotypen Beteuerungen von der Rolle der Schrift, abermals ein Fenster in die damalige Wirklichkeit auftut.

50 So in StUB H, Nr. Ga 5 (= OÖUB II, S. 134-136, Nr. 95).

51 SUB II, S. 686-687, Nr. 508 (= MDC III, S. 571-572, Nr. 1470). 


\section{Fazit}

Wenn abschließend eine Zusammenfassung versucht werden soll, so geschieht dies unter einem mehrfachen Vorbehalt. Zum einen sind noch nicht alle innerhalb von Untersuchungsraum und -zeit verfügbaren Quellen erfasst. Zum zweiten ist abzuwarten, ob analoge Untersuchungen in anderen Territorien vielleicht abweichende Ergebnisse erbringen. Im Übrigen beanspruchen die hier entwickelten Deutungen keine ausschließliche Geltung; es mögen vielerlei Erscheinungen nebeneinander Platz gehabt haben.

Soweit aus der erhaltenen Überlieferung erkennbar, wurden hochmittelalterliche Urkunden überwiegend als Grundlage für spätere Bestätigungen verwendet. Dadurch wurde die Sache selbst in Erinnerung gerufen und Rechtsnachfolger wurden auf die Handlung ihrer Vorgänger festgelegt. Denn das gute Beispiel der Vorgänger galt nur als Vorbild und nicht als unbedingte Verpflichtung, es ihnen gleichzutun. Mit fortgesetzten Bestätigungen gab es für den einmal geschaffenen Rechtszustand auch immer wieder neue Zeugen.

Gegenüber den Bestätigungen spielte die Urkunde als Beweismittel eine vergleichsweise sehr untergeordnete Rolle. Es regierte eben noch der Zeugenbeweis. Die von allem Anfang an angestrebte Landeskundigkeit lässt sich als der sozusagen äußere Schutzwall für einen Rechtszustand begreifen, der Zeugenbeweis als der innere für den Fall, dass es wirklich hart auf hart ging.

Vor diesem Hintergrund ist es auch möglich, jene Arengen, Publikationsformeln und Korroborationen, welche die Schriftlichkeit als einen Garanten wohlerworbener Rechte preisen, nicht unbedingt als Ausdruck einer mehr oder weniger allgemeinen Überzeugung zu sehen. Diese oft und oft wiederholten Beteuerungen könnten für die geistlichen Urkundenschreiber auch bloß ein Instrument gewesen sein, den gegenüber dem Zeugenbeweis noch eher schwachen Urkundenbeweis zu propagieren. Vielleicht ist es diesen (formelhaften) Beteuerungen zuzuschreiben, dass für das Hochmittelalter die Rolle der Urkunden als Beweismittel um einiges höher eingeschätzt wird als ihr vermutlich zukommt.

Jedoch mögen ganz besonders die Traditionsnotizen, aber auch die Siegelurkunden vielfach dazu benützt worden sein, die Erinnerung an die dort aufgezeichneten Handlungen aufzufrischen oder gar erst zu erzeugen, und das heißt wohl auch: Zeugen für eine Aussage zu „präparieren“. Im Einzelfall wird das sogar ausdrücklich so gesagt. Inwieweit diese Sichtweise verallgemeinert werden darf, lässt sich zumindest derzeit nicht sagen. Vielleicht hängt es mit einem solchen Zweck zusammen, dass in manchen Traditionsbüchern auf die Zeugennamen verzichtet worden ist.

Die Urkunde war noch nicht das gängige Beweismittel, aber hinter vielen Zeugenaussagen wird urkundlich gestützte Erinnerung gestanden sein. 


\section{Listiny a svědkové v právním životě vrcholného středověku}

Studie se věnuje problematice užívání listin ve vrcholném středověku, a sice nikoliv na základě studia listinné produkce, ale na základě formálních i konkrtétních výpovědí v listinách samotných. Zkoumané listiny pocházejí především z Rakouska a Slovinska, vedle toho také z Furlanska a Istrie. Časově se výzkum zaměřuje na 11. a 12. století. Tak jako jinde, jsou i zde často potvrzovány panovnické listiny a stejný obraz platí i pro listiny soukromé. Jako důkazní materiál u soudu jsou zde naproti tomu listiny doloženy jen zřídka. Vydavatelé se především obávali, že by jejich právní nástupci neuznávali jimi vydané listiny. V listinách často zdůrazňovaný dobrý př́klad předků či předchůdců v úřadu byl totiž sice chvályhodným vzorem, pro právní nástupce však nebyl nikterak závazný. Potvrzení listin pojištovala trvalost právních úkonů a k tomu produkovala nové svědky. „Všeobecná známost“ právního jednání nebyla podstatná teprve po vymření svědků, ale byla zamýšlena již od počátku. Tato „všeobecná známost“ mohla poskytovat určitou ochranu, ale spíše proti zapomění; proti úmyslným narušitelům práva pomáhali jen svědkové. Listina tedy pojištovala spíše pamět na právní počin, než právní počin jako takový. Listiny samy stále znovu zdůrazňují svưj význam pro věčnou pamět, důkazní funkce v nich není zmiňována. V listinách často zdůrazňovaný význam písma pro trvalost právních počinů je zřejmě především propagandou duchovních písařu listin. Svědkové snad čerpali jejich povědomí o právním jednání daleko častěji z listinných pořízení, než to ojediněle můžeme doložit v pramenech. 